\title{
Phytochemical Study of Hedychium malayanum (Zingiberaceae)
}

(Kajian Fitokimia Hedychium malayanum (Zingiberaceae) )

\author{
MOHAMMED YAHYA ABDO, WAN YAACOB WAN AHMAD*, \\ LAILY BIN DIN \& NAZLINA IBRAHIM
}

\begin{abstract}
A phytochemical study was conducted on the stems and leaves of Hedychium malayanum (Zingiberaceae). Three steroids namely stigmasterol (1), sitostenone (2) and stigmast-4-ene-3,6-dione (3) as well as one triterpene, lupenone (4) and one oxygenated sesquiterpene, caryophyllene oxide (5) were successfully isolated from the respective stems and leaves, utilizing several chromatographic techniques. Their structures were elucidated by spectroscopic means (IR, MS, NMR), and by comparison with the literature data.
\end{abstract}

Keywords: Hedychium malayanum; NMR; steroids; terpenoids; Zingiberaceae

ABSTRAK

Kajian fitokimia dijalankan terhadap batang dan daun Hedychium malayanum (Zingiberaceae). Tiga steroid stigmasterol (1), sitostenon (2) dan stigmast-4-ena-3,6-dion (3) berserta triterpena, lupenon (4) dan seskuiterpena beroksigen, kariofilena oksida (5) berjaya dipisahkan masing-masing daripada batang dan daun, menggunakan beberapa teknik kromatografi. Struktur mereka dikenal pasti dengan cara spektroskopi (IM, SJ, RMN) dan secara perbandingan dengan data kepustakaan.

Kata kunci: Hedychium malayanum; RMN; steroid; terpenoid; Zingiberaceae

\section{INTRODUCTION}

Most of Hedychium species are well known for their terpenes. To date, eight Hedychium have been subjected to phytochemical investigations namely $H$. coronarium (Chen et al. 2013), H. gardnerianum (Kumrit et al. 2010), $H$. spicatum (Reddy et al. 2009), H. chrysoleucum (Luo et al. 2009), H.forrestii (Liu et al. 2004), H. villosum (Xiao et al. 2001), H. yunnanense ( $\mathrm{Li}$ et al. 2016) and H. longipetalum (Zhao et al. 2015) and all of them were found to contain labdane diterpenes. Many sesquiterpenoids identified from the essential oils such as cryptomeridiol, $\beta$-eudesmol, oplopanone, elemol, oplodiol, caryophyllene oxide, transnerolidol and $\alpha$-eudesmol were also chromatographically isolated from the $H$. spicatum (Suresh et al. 2013), $H$. coronarium (Kiem et al. 2011), H. chrysoleucum (Luo et al. 2009), H. gardnerianum (Carvalho et al. 2003) and $H$. yunnanense (Zhao et al. 1995). Lupeol as a tritepenoid was isolated once from Hedychium i.e. H. spicatum (Sravani et al. 2012).

The availability of steroids in Hedychium species comes in second after terpenoids. They are distributed in five plants namely H. spicatum (Sravani et al. 2014, 2012), H. coronarium (Kiem et al. 2011), H. chrysoleucum (Luo et al. 2009), H. forrestii (Liu et al. 2004) and $H$. villosum (Xiao et al. 2001). Xanthones and flavonoids which have similar structures can be found in Hedychium gardnerianum (Carvalho et al. 2003; De Medeiros et al. 2008). There are several reports on the compounds isolated from some Hedychium species, but diarylheptanoids in particular were absent except in H. coronarium (Lin et al . 2015). To the best of our knowledge, no phytochemical study has been carried out on the H. malayanum extracts. Therefore, in this paper, we report the isolation and identification of stigmasterol, sitostenone, stigmast-4ene-3,6-dione, lupenone and caryophyllene oxide from the stems and leaves of H. malayanum.

\section{MATERIALS AND METHODS}

The stems and leaves of Hedychium malayanum were collected in December 2013 from Fraser Hills, Pahang, Malaysia. A voucher specimen of WAY 538 was deposited at the Universiti Kebangsaan Malaysia Herbarium (UKMB).

Air-dried ground stems of Hedychium malayanum (420 g) were soaked in $n$-hexane at room temperature for six days to give $1.6 \mathrm{~g}(0.38 \%)$ of a green gummy extract after solvent removal using a rotary evaporator. The extract was subjected to column chromatography (CC) using silica gel (Merck 9385) by eluting with $n$-hexane containing increasing percentages of chloroform. The collected fractions were combined according to their silica gel thin layer chromatography (TLC) (Merck 5554) profiles to yield five fractions (A-E). Fractions B $(80 \mathrm{mg})$ and D (91 $\mathrm{mg}$ ) were separately rechromatographed by preparative thin layer chromatography (PTLC) over silica gel (Merck 7749) to produce compound 1 (6.4 mg) and 
3 (4.6 mg), respectively. Fraction C (132 g) underwent silica gel radial chromatography (RC) (Merck 7749) using increasing polarity of $n$-hexane:ethyl acetate by $10 \%$ to afford compound 2 (4.2 $\mathrm{mg})$.

A $480 \mathrm{~g}$ of air-dried leaves of Hedychium malayanum were macerated with $n$-hexane for six days at room temperature. After filtration and evaporation, the greenish black residue $(5.4 \mathrm{~g}, 1.1 \%)$ was fractionated utilizing vacuum liquid chromatography (VLC) (Merck 7747), analysed by TLC and combined into three fractions (A, $\mathrm{B}$ and $\mathrm{C}$ ) for further separation. The VLC was run on silica gel by using increasing polarity of $n$-hexane-ethyl acetate. Fraction C (66 mg) was subjected to PTLC to yield compound 4 (12.3 mg).

Air-dried ground leaves of Hedychium malayanum $(160 \mathrm{~g})$ were consecutively extracted using solvents of increasing polarity from $n$-hexane to chloroform to ethyl acetate to acetone and lastly methanol (3 days for each). The methanol filtrate was concentrated using a rotary evaporator to yield $3.3 \mathrm{~g}(2.1 \%)$ of green extract. A 2.5 $\mathrm{g}$ portion of the extract was subjected to CC using $10 \%$ polarity increment from 90:10 chloroform-methanol to $100 \%$ methanol. The collected fractions that showed similar TLC profile were combined to yield three fractions I-III. Fraction II (189 mg) was subjected to RC by eluting with 90:10 of $n$-hexane-ethyl acetate in $10 \%$ polarity increment to produce other three sub-fractions. The subfraction 3 was then fractionated over silica gel on PTLC to give compound $\mathbf{5}$ (11.1 mg).

\section{RESULTS AND DISCUSSION}

The NMR spectral data $\left({ }^{1} \mathrm{H}\right.$ and ${ }^{13} \mathrm{C}$ APT $)$ of stigmasterol (Table 1), sitostenone (Table 2) and stigmast-4-ene-3,6-

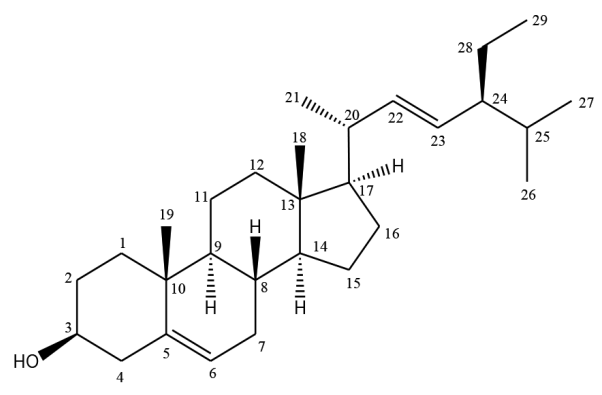

Stigmasterol (1)

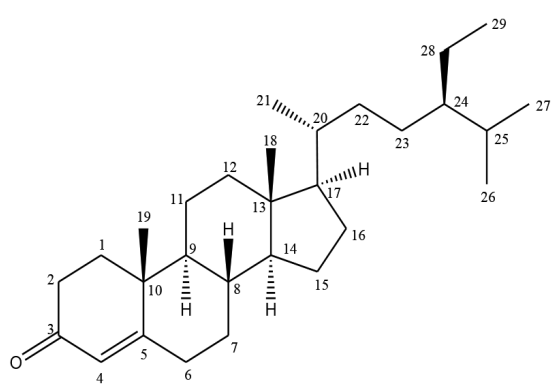

Sitostenone (2)<smiles></smiles>

Stigmast-4-ene-3,6-dione (3)

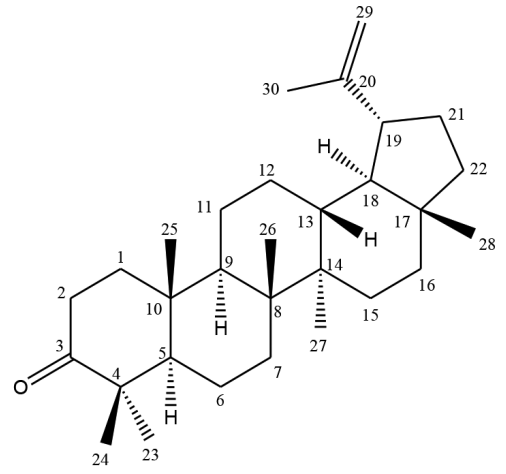

Lupenone (4)<smiles>C=C1CC2O[C@]2(C)CC[C@@H]2[C@@H]1CC2(C)C</smiles>

Caryophyllene oxide (5) 
TABLE $1 .{ }^{1} \mathrm{H}$ and ${ }^{13} \mathrm{C}$ APT NMR data of compound $\mathbf{1}$ and $*$ literature $\left(\mathrm{CDCl}_{3}, 600 \mathrm{MHz}\right)$

\begin{tabular}{|c|c|c|c|c|}
\hline Position & $\begin{array}{c}\delta_{\mathrm{H}} \mathrm{ppm} \text { (number of protons, } \\
\text { multiplicity, } J \mathrm{~Hz} \text { ) }\end{array}$ & $\begin{array}{c}* \delta_{\mathrm{H}} \text { ppm (number of protons, } \\
\text { multiplicity, } J \mathrm{~Hz} \text { ) }\end{array}$ & $\delta_{\mathrm{C}} \mathrm{ppm}$ & $* \delta_{\mathrm{C}} \mathrm{ppm}$ \\
\hline 1 & & & 37.3 & 37.2 \\
\hline 2 & & & 31.6 & 31.6 \\
\hline 3 & $3.53(1 \mathrm{H}, m)$ & $3.51(1 \mathrm{H}, m)$ & 71.8 & 71.8 \\
\hline 4 & & & 42.3 & 42.3 \\
\hline 5 & & & 140.8 & 140.7 \\
\hline 6 & $5.35(1 \mathrm{H}, b d, J=3.0)$ & $5.35(1 \mathrm{H}, m)$ & 121.8 & 121.7 \\
\hline 7 & & & 32.0 & 31.9 \\
\hline 8 & & & 31.9 & 31.9 \\
\hline 9 & & & 50.1 & 50.1 \\
\hline 10 & & & 36.5 & 36.5 \\
\hline 11 & & & 21.0 & 21.1 \\
\hline 12 & & & 39.7 & 39.7 \\
\hline 13 & & & 42.2 & 42.3 \\
\hline 14 & & & 56.9 & 56.9 \\
\hline 15 & & & 24.4 & 24.3 \\
\hline 16 & & & 29.0 & 28.9 \\
\hline 17 & & & 55.9 & 55.9 \\
\hline 18 & $0.70(3 \mathrm{H}, s)$ & $0.67(3 \mathrm{H}, s)$ & 12.1 & 12.0 \\
\hline 19 & $1.01(3 \mathrm{H}, s)$ & $1.00(3 \mathrm{H}, s)$ & 19.4 & 19.4 \\
\hline 20 & & & 40.5 & 40.5 \\
\hline 21 & $0.92(3 \mathrm{H}, d, J=6.6)$ & $0.92(3 \mathrm{H}, d, J=6.0)$ & 21.1 & 21.1 \\
\hline 22 & $5.15(1 \mathrm{H}, d d, J=8.4,15.0)$ & $5.14(1 \mathrm{H}, d d, J=6.5,15.0)$ & 138.4 & 138.3 \\
\hline 23 & $5.02(1 \mathrm{H}, d d, J=9.0,15.0)$ & $5.04(1 \mathrm{H}, d d, J=9.0,15.0)$ & 129.3 & 129.3 \\
\hline 24 & & & 51.3 & 51.2 \\
\hline 25 & & & 31.9 & 31.9 \\
\hline 26 & $0.85(3 \mathrm{H}, d, J=6.0)$ & $0.84(3 \mathrm{H}, d, J=6.0)$ & 21.2 & 21.2 \\
\hline 27 & $0.80(3 \mathrm{H}, d, J=6.0)$ & $0.80(3 \mathrm{H}, d, J=6.0)$ & 19.0 & 19.0 \\
\hline 28 & & & 25.4 & 25.4 \\
\hline 29 & $0.82(3 \mathrm{H}, t, J=7.5)$ & $0.82(3 \mathrm{H}, t, J=6.5)$ & 12.3 & 12.2 \\
\hline
\end{tabular}

TABLE $2 .{ }^{1} \mathrm{H}$ and ${ }^{13} \mathrm{C}$ APT NMR data of compound 2 and *literature $\left(\mathrm{CDCl}_{3}, 600 \mathrm{MHz}\right)$

\begin{tabular}{|c|c|c|c|c|}
\hline Position & $\begin{array}{c}\delta_{\mathrm{H}} \mathrm{ppm} \text { (number of protons, } \\
\text { multiplicity, } J \mathrm{~Hz} \text { ) }\end{array}$ & $\begin{array}{c}* \delta_{\mathrm{H}} \text { ppm (number of protons, } \\
\text { multiplicity, } J \mathrm{~Hz} \text { ) }\end{array}$ & $\delta_{\mathrm{C}} \mathrm{ppm}$ & $* \delta_{\mathrm{C}} \mathrm{ppm}$ \\
\hline 1 & & & 35.7 & 35.7 \\
\hline 2 & & & 34.0 & 34.0 \\
\hline 3 & & & 199.8 & 199.7 \\
\hline 4 & $5.73(1 \mathrm{H}, s)$ & $5.72(1 \mathrm{H}, s)$ & 123.7 & 123.7 \\
\hline 5 & & & 171.9 & 171.8 \\
\hline 6 & & & 33.0 & 32.9 \\
\hline 7 & & & 32.1 & 32.0 \\
\hline 8 & & & 35.6 & 35.6 \\
\hline 9 & & & 53.8 & 53.8 \\
\hline 10 & & & 38.6 & 38.6 \\
\hline 11 & & & 21.0 & 21.0 \\
\hline 12 & & & 39.6 & 39.6 \\
\hline 13 & & & 42.4 & 42.4 \\
\hline 14 & & & 55.9 & 55.8 \\
\hline 15 & & & 28.2 & 28.2 \\
\hline 16 & & & 24.2 & 24.2 \\
\hline 17 & & & 56.0 & 55.9 \\
\hline 18 & $0.72(3 \mathrm{H}, s)$ & $0.71(3 \mathrm{H}, s)$ & 11.9 & 11.9 \\
\hline 19 & $1.19(3 \mathrm{H}, s)$ & $1.18(3 \mathrm{H}, s)$ & 17.4 & 17.4 \\
\hline 20 & & & 36.1 & 36.1 \\
\hline 21 & $0.92(3 \mathrm{H}, d, J=6.6)$ & $0.92(3 \mathrm{H}, d, J=6.5)$ & 18.7 & 18.7 \\
\hline 22 & & & 33.9 & 33.8 \\
\hline 23 & & & 26.0 & 26.0 \\
\hline 24 & & & 29.1 & 29.1 \\
\hline 25 & & & 45.8 & 45.8 \\
\hline 26 & $0.83(3 \mathrm{H}, d, J=6.6)$ & $0.83(3 \mathrm{H}, d, J=6.5)$ & 19.9 & 19.8 \\
\hline 27 & $0.82(3 \mathrm{H}, d, J=7.2)$ & $0.81(3 \mathrm{H}, d, J=6.8)$ & 19.0 & 19.0 \\
\hline 28 & & & 23.1 & 23.0 \\
\hline 29 & $0.85(3 \mathrm{H}, t, J=7.2)$ & $0.85(3 \mathrm{H}, t, J=6.7)$ & 12.0 & 11.9 \\
\hline
\end{tabular}


dione (Table 3 ) were in agreement with those of DeEknamkul and Potduang (2003)/Hussain et al. (2008), Pardo et al. (2000) and Seca et al. (2000), respectively. Stigmasterol was reported previously from other two Hedychium species of H.forrestii (Liu et al. 2004) and $H$. coronarium (Kiem et al. 2011) and also found in many other plant families including Zingiberaceae such as Renealmia (Lognay et al. 1989), Alpinia (Le et al. 2007; Phan \& Phan 2004; Phan et al. 2012, 2007, 2005), Zingiber (Peng et al. 2007), Etlingera (Mahdavi 2014; Yahya et al. 2011) and Curcuma (Shamim et al. 2011). To the best of our knowledge, this is the first time sitostenone and stigmast-4-ene-3,6-dione have been isolated from genus Hedychium, which contributes to the database of steroids from Hedychium. Additionally, these two steroids have never been reported previously from any other genus of Zingiberaceae except in Etlingera (Mohamad et al. 2005).

Comparison of the ${ }^{1} \mathrm{H}$ and ${ }^{13} \mathrm{C}$ APT NMR spectral data of lupenone (Table 4) with the previously published data of Prachayasittikul et al. (2010) confirmed that both are of the same compound. Based on literature review, triterpenoid lupenone was isolated for the first time from Zingiberaceae.
In fact, the presence of other triterpenoids in Zingiberaceae species of Curcuma longa (Mohamed et al.2003), Globba racemosa (Qiao et al. 1999), Renealmia alpinia (Lognay et al. 1989) and Hedychium gardnerianum (Saleh et al. 1981) supports its existence in Hedychium malayanum.

The similarity of the ${ }^{1} \mathrm{H}$ and ${ }^{13} \mathrm{C}$ APT NMR spectral data of caryophyllene oxide (Table 5) with those of the published data (Orihara et al. 1994) confirmed that both are of the same structure. Besides frequent identification of the sesquiterpene caryophyllene oxide as a major compound in essential oils from many Hedychium species, it was also purified from H. spicatum (Suresh et al. 2013) and Alpinia conchigera (Aziz et al. 2013).

\section{CONCLUSION}

The isolation and characterization of stigmasterol (1), sitostenone (2), stigmast-4-ene-3,6-dione (3), lupenone (4) and caryophyllene oxide (5) from the stems and leaves of Hedychium malayanum were the first ever to be reported from this plant. This is the first report of sitostenone, stigmast-4-ene-3,6-dione, lupenone from Hedychium

TABLE $3 .{ }^{1} \mathrm{H}$ and ${ }^{13} \mathrm{C}$ APT NMR data of compound $\mathbf{3}$ and *literature $\left(\mathrm{CDCl}_{3}, 600 \mathrm{MHz}\right)$

\begin{tabular}{|c|c|c|c|c|}
\hline Position & $\begin{array}{c}\delta_{\mathrm{H}} \mathrm{ppm} \text { (number of protons, } \\
\text { multiplicity, } J \mathrm{~Hz})\end{array}$ & $\begin{array}{c}* \delta_{\mathrm{H}} \text { ppm (number of protons, } \\
\text { multiplicity, } J \mathrm{~Hz})\end{array}$ & $\delta_{\mathrm{C}} \mathrm{ppm}$ & $* \delta_{\mathrm{C}} \mathrm{ppm}$ \\
\hline 1 & & & 35.5 & 35.5 \\
\hline 2 & & & 34.0 & 34.0 \\
\hline 3 & & & 199.6 & 199.5 \\
\hline 4 & $6.18(1 \mathrm{H}, s)$ & $6.17(1 \mathrm{H}, s)$ & 125.5 & 125.4 \\
\hline 5 & & & 161.1 & 161.1 \\
\hline 6 & & & 202.4 & 202.4 \\
\hline 7 & $2.69(1 \mathrm{H}, d d, J=4.2,16.2)$ & $2.68(1 \mathrm{H}, d d, J=3.7,15.6)$ & 46.9 & 46.8 \\
\hline 8 & & & 34.2 & 34.2 \\
\hline 9 & & & 51.0 & 51.0 \\
\hline 10 & & & 39.8 & 39.8 \\
\hline 11 & & & 20.9 & 20.9 \\
\hline 12 & & & 39.1 & 39.1 \\
\hline 13 & & & 42.5 & 42.5 \\
\hline 14 & & & 56.7 & 56.5 \\
\hline 15 & & & 24.0 & 24.0 \\
\hline 16 & & & 28.0 & 28.0 \\
\hline 17 & & & 55.8 & 55.8 \\
\hline 18 & $0.73(3 \mathrm{H}, s)$ & $0.72(3 \mathrm{H}, s)$ & 11.9 & 11.9 \\
\hline 19 & $1.17(3 \mathrm{H}, s)$ & $1.17(3 \mathrm{H}, s)$ & 17.5 & 17.5 \\
\hline 20 & & & 36.1 & 36.0 \\
\hline 21 & $0.94(3 \mathrm{H}, d, J=6.6)$ & $0.93(3 \mathrm{H}, d, J=6.4)$ & 18.7 & 18.7 \\
\hline 22 & & & 33.8 & 33.8 \\
\hline 23 & & & 26.0 & 26.0 \\
\hline 24 & & & 29.1 & 29.1 \\
\hline 25 & & & 45.8 & 45.8 \\
\hline 26 & $0.82(3 \mathrm{H}, d, J=6.6)$ & $0.81(3 \mathrm{H}, d, J=6.5)$ & 19.0 & 19.0 \\
\hline 27 & $0.83(3 \mathrm{H}, d, J=7.2)$ & $0.84(3 \mathrm{H}, d, J=6.5)$ & 19.9 & 19.8 \\
\hline 28 & & & 23.1 & 23.0 \\
\hline 29 & $0.85(3 \mathrm{H}, t, J=7.2)$ & $0.85(3 \mathrm{H}, t, J=6.7)$ & 12.0 & 12.0 \\
\hline
\end{tabular}


TABLE 4. ${ }^{1} \mathrm{H}$ and ${ }^{13} \mathrm{C}$ APT NMR data of compound 4 and * literature $\left(\mathrm{CDCl}_{3}, 600 \mathrm{MHz}\right)$

\begin{tabular}{|c|c|c|c|c|}
\hline Position & $\begin{array}{c}\delta_{\mathrm{H}} \mathrm{ppm} \text { (number of protons, } \\
\text { multiplicity, } J \mathrm{~Hz})\end{array}$ & $\begin{array}{c}* \delta_{\mathrm{H}} \text { ppm (number of protons, } \\
\text { multiplicity, } J \mathrm{~Hz} \text { ) }\end{array}$ & $\delta_{\mathrm{C}} \mathrm{ppm}$ & $* \delta_{\mathrm{C}} \mathrm{ppm}$ \\
\hline 1 & & & 39.6 & 39.6 \\
\hline 2 & & & 34.2 & 34.1 \\
\hline 3 & & & 218.4 & 218.2 \\
\hline 4 & & & 47.4 & 47.3 \\
\hline 5 & & & 54.9 & 54.9 \\
\hline 6 & & & 19.7 & 19.2 \\
\hline 7 & & & 33.6 & 33.5 \\
\hline 8 & & & 40.8 & 40.7 \\
\hline 9 & & & 49.8 & 49.7 \\
\hline 10 & & & 36.9 & 36.8 \\
\hline 11 & & & 21.5 & 21.4 \\
\hline 12 & & & 25.1 & 25.1 \\
\hline 13 & & & 38.2 & 38.1 \\
\hline 14 & & & 42.9 & 42.8 \\
\hline 15 & & & 27.4 & 27.4 \\
\hline 16 & & & 35.5 & 35.5 \\
\hline 17 & & & 43.0 & 42.9 \\
\hline 18 & & & 48.2 & 48.2 \\
\hline 19 & & & 48.0 & 47.9 \\
\hline 20 & & & 150.9 & 150.8 \\
\hline 21 & & & 29.7 & 29.6 \\
\hline 22 & & & 40.0 & 39.9 \\
\hline 23 & $1.08(3 \mathrm{H}, s)$ & $1.04(3 \mathrm{H}, s)$ & 26.7 & 26.6 \\
\hline 24 & $1.03(3 \mathrm{H}, s)$ & $1.00(3 \mathrm{H}, s)$ & 21.1 & 21.0 \\
\hline 25 & $0.94(3 \mathrm{H}, s)$ & $0.90(3 \mathrm{H}, s)$ & 16.0 & 15.9 \\
\hline 26 & $1.26(3 \mathrm{H}, s)$ & $1.22(3 \mathrm{H}, s)$ & 15.8 & 15.7 \\
\hline 27 & $0.96(3 \mathrm{H}, s)$ & $0.93(3 \mathrm{H}, s)$ & 14.5 & 14.4 \\
\hline 28 & $0.80(3 \mathrm{H}, s)$ & $0.77(3 \mathrm{H}, s)$ & 18.0 & 17.9 \\
\hline 29 & $4.58(1 \mathrm{H}, b s, \mathrm{H}-29 \mathrm{a})$ & $4.55(1 \mathrm{H}, b s, \mathrm{H}-29 \mathrm{a})$ & 109.4 & 109.3 \\
\hline \multirow[t]{2}{*}{30} & $4.70(1 \mathrm{H}, b s, \mathrm{H}-29 \mathrm{~b})$ & $4.66(1 \mathrm{H}, b s, \mathrm{H}-29 \mathrm{~b})$ & 20.3 & 19.6 \\
\hline & $1.69(3 \mathrm{H}, s)$ & $1.66(3 \mathrm{H}, s)$ & & \\
\hline
\end{tabular}

TABLE 5. ${ }^{1} \mathrm{H}$ and ${ }^{13} \mathrm{C}$ APT NMR data of compound $\mathbf{5}$ and $*$ literature $\left(\mathrm{CDCl}_{3}, 600 \mathrm{MHz}\right)$

\begin{tabular}{ccccc}
\hline Position & $\begin{array}{c}\delta_{\mathrm{H}} \text { ppm (number of protons, } \\
\text { multiplicity }, J \mathrm{~Hz})\end{array}$ & $\begin{array}{c}* \delta_{\mathrm{H}} \mathrm{ppm}(\text { number of protons, } \\
\text { multiplicity }, J \mathrm{~Hz})\end{array}$ & $\delta_{\mathrm{C}} \mathrm{ppm}$ & $* \delta_{\mathrm{C}} \mathrm{ppm}$ \\
\hline 1 & $1.76(1 \mathrm{H}, t, J=9.9)$ & $1.76(1 \mathrm{H}, d d, J=10.1,4.2)$ & 50.7 & 50.8 \\
2 & & & 27.2 & 27.4 \\
3 & & & 39.1 & 39.3 \\
4 & $2.88(1 \mathrm{H}, d d, J=10.8,4.2)$ & $2.86(1 \mathrm{H}, d d, J=10.6,4.2)$ & 59.9 & 60.0 \\
6 & & & 63.8 & 63.9 \\
7 & & & 30.2 & 30.4 \\
8 & & & 29.8 & 29.9 \\
9 & & & 151.8 & 152.0 \\
10 & $2.62(1 \mathrm{H}, q, J=9.6)$ & 48.7 & 48.9 \\
11 & & $1.20(3 \mathrm{H}, s)$ & 39.8 & 39.9 \\
12 & & & 34.0 & 34.2 \\
13 & $1.20(3 \mathrm{H}, s)$ & 17.0 & 17.2 \\
14 & $4.86(1 \mathrm{H}, b d, J=1.2, \mathrm{H}-14 \mathrm{a})$ & $4.86(1 \mathrm{H}, d, J=1.2, \mathrm{H}-14 \mathrm{a})$ & 112.8 & 113.0 \\
& $4.97(1 \mathrm{H}, b d, J=0.6, \mathrm{H}-14 \mathrm{~b})$ & $4.97(1 \mathrm{H}, d, J=1.2, \mathrm{H}-14 \mathrm{~b})$ & & \\
15 & $1.00(3 \mathrm{H}, s)$ & $1.00(3 \mathrm{H}, s)$ & 29.9 & 30.1 \\
16 & $0.98(3 \mathrm{H}, s)$ & $0.98(3 \mathrm{H}, s)$ & 21.6 & 21.8 \\
\hline
\end{tabular}


genus and lupenone from Zingiberaceae family. The work was done using several chromatographic techniques and spectroscopic analyses.

\section{ACKNOWLEDGEMENTS}

We would like to express our gratitude to the School of Chemical Sciences and Food Technology, Universiti Kebangsaan Malaysia for the provision of research facilities and technical assistance. We also wish to thank the Ministry of Higher Education Malaysia and Universiti Kebangsaan Malaysia for the financial support through the Fundamental Research Grant Scheme of UKM-ST06-FRGS0110-2009 and Publication Intensification Fund of DLP-2013-018.

\section{REFERENCES}

Aziz, A.N., Ibrahim, H., Syamsir, D.R., Mohtar, M., Vejayan, J. \& Awang, K. 2013. Antimicrobial compounds from Alpinia conchigera. Journal of Ethnopharmacology 145: 798-802.

Carvalho, M.J., Carvalho, L.M., Ferreira, A.M. \& Silva, A.M. 2003. A new xanthone from Hedychium gardnerianum. Natural Product Research 17(6): 445-449.

Chen, J.J., Ting, C.W., Wu, Y.C., Hwang, T.L., Cheng, M.J., Sung, P.J., Wang, T.C. \& Chen, J.F. 2013. New labdanetype diterpenoids and anti-inflammatory constituents from Hedychium coronarium. International Journal of Molecular Sciences 14(7): 13063-13077.

De Medeiros, J.M.R., Medeiros, H. \& Mascarenhas, C. 2008. Flavonoid anticoagulant agents extracted from Hedychium gardnerianum. Port. Pat. Appl. PT 103685 A 20080909.

De-Eknamkul, W. \& Potduang, B. 2003. Biosynthesis of $\beta$-sitosterol and stigmasterol in Croton sublyratus proceeds via a mixed origin of isoprene units. Phytochemistry 62(3): 389-398.

Hussain, M.M., Rahman, M.S., Jabbar, A. \& Rashid, M.A. 2008. Phytochemical and biological investigations of Albizzia lebbeck Benth. Boletin Latinoamericano y del Caribe de Plantas Medicinales y Aromáticas 7(5): 273-278.

Kiem, P.V., Thuy, N.T.K., Anh, H.L.T., Nhiem, N.X., Minh, C.V., Yen, P.H., Ban, N.K., Hang, D.T., Tai, B.H., Tuyen, N.V., Mathema, V.B., Koh, Y.S. \& Kim, Y.H. 2011. Chemical constituents of the rhizomes of Hedychium coronarium and their inhibitory effect on the pro inflammatory cytokines production LPS-stimulated in bone marrow-derived dendritic cells. Bioorganic \& Medicinal Chemistry Letters 21(24): 7460-7465.

Kumrit, I., Suksamrarn, A., Meepawpan, P., Songsri, S. \& Nuntawong, N. 2010. Labdane-type diterpenes from Hedychium gardnerianum with potent cytotoxicity against human small cell lung cancer cells. Phytotherapy Research 24(7): 1009-1013.

Le, H.T., Phan, M.G. \& Phan, T.S. 2007. Biologically active phenolic constituents from Alpinia gagnepainii K. Schum. Tap Chi Hoa Hoc 45(1): 126-130.

Li, Y.P., Zhao, S.M., Xu, J.J., Zeng, G.Z., Li, Y., Tan, N.H. \& Yang, Y. 2016. New labdane diterpenes from Hedychium yunnanense with cytotoxicity and inhibitory effects on nitric oxide production. Natural Product Research 30(23): 2669-2674.
Lin, Y.S., Lin, J.H., Chang, C.C. \& Lee, S.S. 2015. Tetrahydropyranand tetrahydrofuran-containing diarylheptanoids from Hedychium coronarium rhizomes. Journal of Natural Products 78(2): 181-187.

Liu, L.J., Guo, W.C., Peng, Q.L., Yan, S.J., Su, J.Y. \& Zeng, L.M. 2004. Studies of the chemical constituents of Hedychium forrestii Diels. Zhongshan Daxue Xuebao, Ziran Kexueban 43(5): 58-60.

Lognay, G., Marlier, M., Haubruge, E., Trevejo, E. \& Severin, M. 1989. Study of the lipids from Renealmia alpinia (Rott) Maas. Grasas y Aceites 40(6): 351-355.

Luo, M., Lei, L. \& Lin, H. 2009. Studies on chemical constituents from rhizomes of Hedychium chrysoleucum. Zhongguo Zhong yao za zhi= Zhongguo zhongyao zazhi= China Journal of Chinese Materia. Medica. 34(2): 180-182.

Mahdavi, B. 2014. Chemical constituents of the aerial parts of Etlingera brevilabrum (Zingiberaceae). Pharma Chemica 6(2): 360-365.

Mohamad, H., Lajis, N.H., Abas, F., Ali, A.M., Sukari, M.A., Kikuzaki, H. \& Nakatani, N. 2005. Antioxidative constituents of Etlingera elatior. Journal of Natural Products 68(2): 285-288.

Mohamed, S.M., El-Gengaihi, S.E. \& Motawe, H.M. 2003. Terpenoid from Curcuma longa. Egyptian Journal of Pharmaceutical Sciences 43(1-2): 139-151.

Orihara, Y., Saiki, K. \& Furuya, T. 1994. Biotransformation of caryophyllene oxide by cultured cells of Eucalyptus perriniana. Phytochemistry 35(3): 635-639.

Pardo, F., Perich, F., Torres, R. \& Delle Monache, F. 2000. Stigmast-4-ene-3,6-dione an unusual phytotoxic sterone from the roots of Echium vulgare L. Biochemical Systematics and Ecology 28: 911-913.

Peng, W., Zhang, Y., Yang, K. \& Xiao, Y. 2007. Chemical constituents of Zingiber officinale (Zingiberaceae). Yunnan Zhiwu Yanjiu 29(1): 125-128.

Phan, M.G. \& Phan, T.S. 2004. Phytochemical investigation of Alpinia globosa (Lour.) Horaninov, Zingiberaceae. Tap Chi Hoa Hoc 42(3): 376-378.

Phan, M.G., Dang, T.T.A., Le, H.T. \& Phan, T.S. 2012. Diarylheptanoids and flavanones from Alpinia napoensis. Tap Chi Hoa Hoc 50(5): 537-541.

Phan, M.G., Le, H.T., Phan, T.S. \& Otsuka, H. 2007. Chemical constituents of the fruits of Alpinia conchigera Griff. (Zingiberaceae). Tap Chi Hoa Hoc 45(4): 509-512.

Phan, M.G., Dang, B.T. \& Phan, T.S. 2005. Flavonoid compounds from the rhizomes of Alpinia conchigera Griff., Zingiberaceae. Tap Chi Hoa Hoc 43(1): 105-108.

Prachayasittikul, S., Saraban, P., Cherdtrakulkiat, R., Ruchirawat, S. \& Prachayasittikul, V. 2010. New bioactive triterpenoids and antimicrobial activity of Diospyros rubralec. Experimental and Clinical Sciences Journal 9: 1-10.

Qiao, C., Xu, L., Shen, Y. \& Hao, X. 1999. Chemical constituents from Globba racemosa Smith. Huaxue Yanjiu Yu Yingyong 11(5): 575-576.

Reddy, P.P., Rao, R.R., Shashidhar, J., Sastry, B.S., Rao, J.M. \& Babu, K.S. 2009. Phytochemical investigation of labdane diterpenes from the rhizomes of Hedychium spicatum and their cytotoxic activity. Bioorganic \& Medicinal Chemistry Letters 19(21): 6078-6081.

Saleh, M.M., Shabana, M.K.S., Guindi, E.R. \& Kamal, R.M. 1981. Phytochemical and molluscicidal study on Hedychium gardnerianum, Wall. as a method for control of schistosoma. 
Int. Conf. Chem. Biotechnol. Act. Nat. Prod. [Proc.], 1st 3(1): 293-297.

Seca, A., Silva, A., Silvestre, A., Cavaleiro, J.A.S., Domingues, F. \& Pascoal Neto, C. 2000. Chemical composition of the light petroleum extract of Hibiscus cannabinus bark and core. Phytochemical Analysis 11: 345-350.

Shamim, A., Mohd, A. \& Husain, A.S. 2011. New aliphatic constituents from the rhizomes of Curcuma oligantha Trimen var. oligantha. International Journal of Research in Ayurveda \& Pharmacy 2(3): 797-800.

Sravani, T., Paarakh, P.M. \& Shruthi, S.D. 2014. In silico and in vitro anthelmintic activity of stigmasterol $\beta$ D-glucoside isolated from rhizomes of Hedychium spicatum Buch. Ham. World Journal of Pharmacy and Pharmaceutical Sciences 3(9): 664-672.

Sravani, T., Paarakh, P.M. \& Vedamurthy, A.B. 2012. Isolation of phytoconstituents from the rhizomes of Hedychium spicatum Buch. Ham. Journal of Pharmacy Research 5(1): 526-527.

Suresh, G., Poornima, B., Babu, K.S., Yadav, P.A., Rao, M.S., Siva, B., Parsad, K.R., Nayak, V.L. \& Ramakrishna, S. 2013. Cytotoxic sesquiterpenes from Hedychium spictum: Isolation, structure elucidation and structure-activity relationship studies. Fitoterapia 86: 100-107.

Xiao, P., Sun, C., Zahid, M., Ishrud, O. \& Pan, Y. 2001. New diterpene from Hedychium villosum. Fitoterapia 72(7): 837-838.
Yahya, M.A.A., Yaacob, W.A. \& Nazlina, I. 2011. Isolation of chemical constituents from rhizomes of Etlingera sphaerocephala var. grandiflora. Malaysian Journal of Analytical Sciences 15(1): 22-26.

Zhao, H., Zeng, G., Zhao, S., Xu, J., Kong, L., Li, Y., Tan, N. \& Yang, S. 2015. Cytotoxic labdane-type diterpenes from Hedychium longipetalum inhibiting production of nitric oxide. Bioorganic \& Medicinal Chemistry Letters 25(20): 4572-4575.

Zhao, Q., Hao, X.J., Chen, Y.Z. \& Zou, C. 1995. Sesquiterpenoids from Hedychium yunnanense. Yunnan Zhiwu Yanjiu 17(2): 201203.

Pusat Pengajian Sains Kimia dan Teknologi Makanan Fakulti Sains \& Teknologi

Universiti Kebangsaan Malaysia

43600 UKM Bangi, Selangor Darul Ehsan

Malaysia

*Corresponding author; email: wanyaa@ukm.edu.my

Received: 26 March 2015

Accepted: 28 April 2016 